\title{
Incorporação de Estratificações Salíferas na Modelagem de Velocidade de uma Jazida da Bacia de Santos
}

Thiago Yamamoto(1), Alexandre Maul(1), Eliane Born(1), Fernanda Gobatto(1), Maria Taryn Campos(1), María Gonzáles(2)

(1)Petrobras (2)Paradigm

Copyright 2016, SBGf - Sociedade Brasileira de Geofísica

Este texto foi preparado para a apresentação no VII Simpósio Brasileiro de Geofísica, Ouro Preto, 25 a 27 de outubro de 2016. Seu conteúdo foi revisado pelo Comitê Técnico do VII SimBGf, mas não necessariamente representa a opinião da SBGf ou de seus associados. E proibida a reprodução total ou parcial deste material para propósitos comerciais sem prévia autorização da SBGf.

\section{Resumo}

In the last decades, geophysicists have developed several techniques to generate better seismic images. Some of them are:

- Better seismic acquisition techniques: acquisition in high definition, multiazimute, nodes etc.

- More robust algorithms of inversion: High resolution tomography and FWI (Full-Waveform Inversion)

- Incorporation of anisotropic parameters: VTI (Vertical transverse isotropy), TTI (Tilted Transverse isotropy) and orthorhombic

- More complex migration algorithms (RTM Reverse Time Migration).

In this work, we present the results obtained from the application of a new way to build velocity models aiming to include the existing stratifications within the evaporite section, in the Santos Basin, Brazil.

\section{Introdução}

As aquisições e processamentos sísmicos para os campos do polo Pré-Sal da Bacia de Santos sempre se ampararam nas melhores técnicas disponíveis no mercado. Apesar de todas as limitações dos métodos e complexidade da geologia local, na medida do possível, boas imagens sísmicas vêm sendo alcançadas.

Entretanto, esses dados ainda precisam de uma amarração adicional com poços pelos intérpretes. Vale mencionar que a diferença entre profundidade prevista dos topos dos reservatórios com a constatada em poços diminui cada vez mais, ficando muitas vezes dentro do limite da resolução sísmica.

Apesar de todos os avanços na área da geofísica a indústria de petróleo, de uma forma geral, cobra desta disciplina uma melhor caracterização dos reservatórios.

Essa exigência se faz necessária para, entre outros motivos, suportar estudos de análise de incertezas, utilizados para avaliar a viabilidade econômica e dar suporte à decisão sobre projetos de desenvolvimento da produção.

Um dos grandes obstáculos para a construção de uma imagem sísmica mais fiel à geologia local é, justamente, o modelo de velocidade intervalar que, geralmente, é concebido por inversões tomográficas. A resolução e qualidade desses modelos são fortemente impactados pela: geologia em subsuperfície, frequência da sísmica e a geometria da aquisição.

Os modelos de velocidades especialmente nos campos do Pré-Sal da Bacia de Santos não contemplam, de uma maneira satisfatória, a estruturação geológica da área. E este problema é mais destacado dentro da seção evaporítica.

O pacote de sal apresenta uma alta complexidade estrutural devido à tectônica salífera (Oliveira et al., 2015), podendo se mencionar: domos salinos, diápiros, mini bacias etc. Além dessa compartimentação a seção evaporítica apresenta uma alta variedade litológica, tais como: halita, anidrita, gipsita, taquidrita, silvita etc. Esses sais possuem um intervalo de velocidades variando de pouco mais de $4000 \mathrm{~m} / \mathrm{s}$ até $6000 \mathrm{~m} / \mathrm{s}$ (informação obtida a partir dos perfis de poços perfurados nos campos).

Uma forma de se aprimorar o modelo de velocidade seria aplicar FWI (Full-Waveform Inversion). Contudo, na época (2013) da contratação do processamento do dado utilizado nesse trabalho não se cogitou usar essa técnica. Mas, com o continuo desenvolvimento computacional e de aquisição sísmica, o FWI vem se tornando cada vez mais difundido e viável. Nas grandes operadoras de petróleo cada vez mais existem grupos internos estudando a aplicação dessa metodologia ou, um grande esforço na viabilização da contratação desta modalidade de processamento. Para maiores detalhes sobre o FWI consulte Virieux \& Operto (2009).

Assim, visando sanar esse problema com o modelo de velocidades, surgiu como alternativa a inclusão de estratificações dentro da seção evaporítica que se sobrepõem aos reservatórios do Pré-Sal, tanto na Bacia de Campos quanto na Bacia de Santos, a partir da resposta sísmica (Maul et al., $(2015,2016)$ e Jardim et al., 2015), González et al. (2016)).

O conhecimento de metodologias de modelagem geológica 3D se torna muito importante na construção dos modelos geológicos de velocidades sísmicas, pois, muitas vezes, variações nas propriedades de rochas e/ou estruturação complexa geram grandes erros na predição das profundidades. Este conceito é fortemente explorado em Maul et al. (2015, 2016), Meneguim et al. (2015) baseado na metodologia proposta por Fetter et al. (2004) in Maul et al., (2016).

Os resultados obtidos, além de propiciarem um excelente estabelecimento de cenários de incertezas para posicionamentos em profundidade do topo do reservatório, vêm gerando importantes insumos para estudos de iluminação, inversões sísmicas, migrações, análises geomecânicas e segurança operacional 


\section{Metodologia/ Problema Investigado}

A metodologia aqui proposta leva em consideração a resposta sísmica proveniente das intercalações litológicas existentes dentro da seção evaporítica, tais como a presença de Halita, Anidrita, Taquidrita, Carnalita, Silvinita e outros evaporitos (Oliveira et al., 2015 e Meneguim et al., 2015), bem como a eventual presença de rochas ígneas (Oliveira et al., 2015).

A técnica é suportada pelos seguintes insumos:

-dados de perfis de poços (sônico e litológico);

-sísmica em tempo e profundidade;

-modelo de velocidade utilizado para migrar o dado;

-cubos de impedâncias e fácies obtidos através de inversões sísmicas.

Primeiramente, faz-se um amplo e minucioso trabalho com as informações dos poços, baseada na metodologia proposta por Amaral et al., (2015). Um controle de qualidade é realizado nos perfis sônico e litológico (edição de spikes, marcadores etc.) e verifica se as informações são condizentes com a área. E depois agrupa os sais em três grandes grupos:

1. Halita, que é a litologia predominante sendo assim considerada como referência (background)

2. Sais de alta: anidritas e gipsitas que possuem velocidades maiores que do background

3. Sais de baixa: taquidrita, carnalitas, silvitas etc, cujas velocidades são menores que a da halita.

A partir dos perfis sônicos, calcula-se a velocidade intervalar, quantifica-se as proporções dos sais e a estatística das velocidades (média, mediana etc.) para os três diferentes grupos descritos acima.

Por último, o modelo de velocidade original ou um modelo de velocidade proporcional ao background (halita) é perturbado pelo intervalo de valores encontrado nos poços. A amplitude e os volumes de impedâncias são usados como guia para a extrapolação dentro da seção evaporítica (Maul et al. 2014, 2016). Amplitudes/ impedâncias altas modelam as elevadas velocidades e as baixas velocidades são construídas pelas baixas amplitudes/ impedâncias;

Para este estudo foram disponibilizados 19 poços de um projeto real situado na Bacia de Santos, compreendendo uma área de, aproximadamente $230 \mathrm{Km}^{2}$. Essa boa proporção de informações (número de poços $X$ área) contribuiu para uma análise estatística mais precisa das ocorrências dos sais e velocidades de cada litotipo existente da seção evaporítica (figura 1, 2, 3 e tabela 1).

Cerca de $90 \%$ do total de litotipos na seção evaporítica estudada é halita (figura 01). Contudo, esse valor muda de poço a poço (figura 02). Nota-se que em nenhum deles a proporção somada dos sais de alta e baixa superam os $20 \%$. A média das velocidades de todos os poços ficou em torno de $4570 \mathrm{~m} / \mathrm{s}$. Na tabela 01, tem-se uma noção melhor das estatísticas das velocidades.

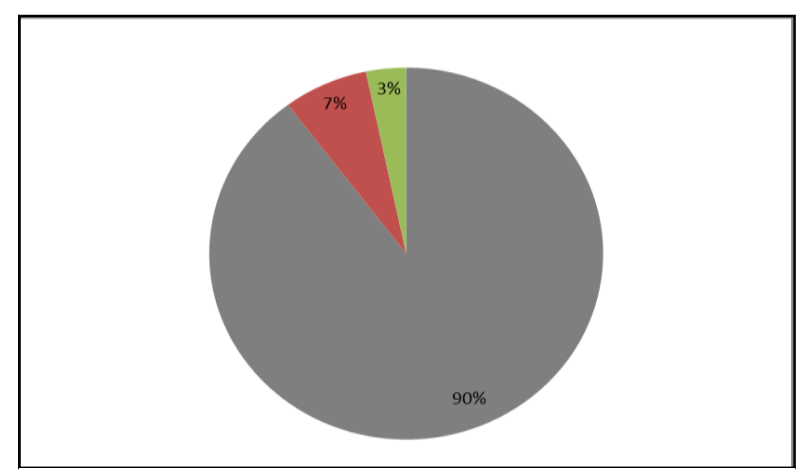

Figura 1 - Proporção de sal(halita em cinza, sais de alta em vermelho e sais de baixa em verde).

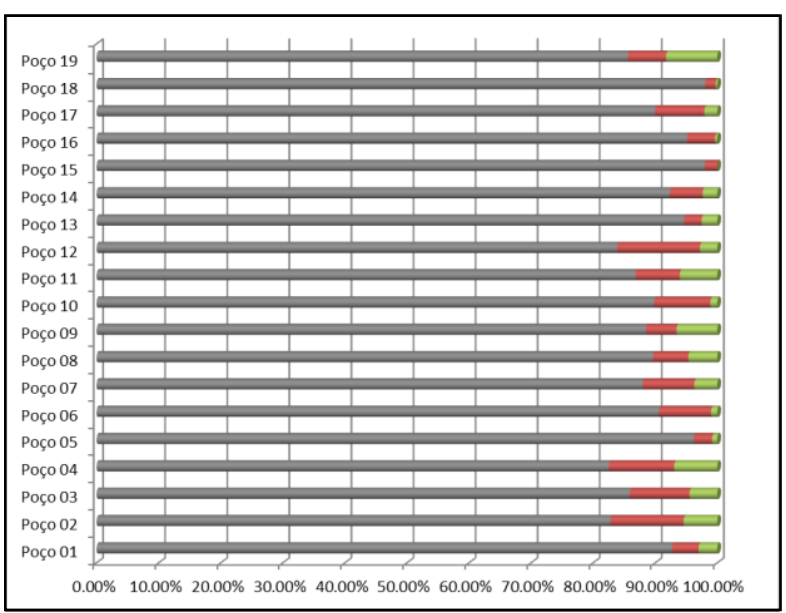

Figura 2 - Proporção de sal (halita em cinza, sais de alta em vermelho e sais de baixa em verde). Notase que a soma dos sais excluindo a halita não ultrapassa os $20 \%$

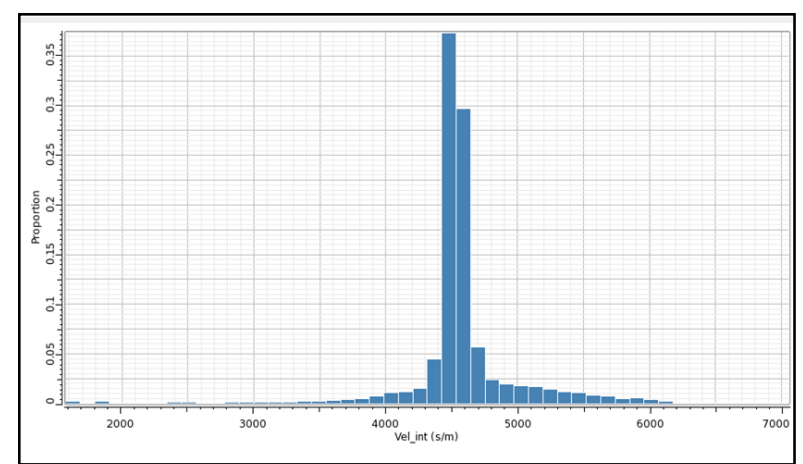

Figura 03 - Histograma das velocidades dos sais 
Tabela 1: Estatística das velocidades do sal.

\begin{tabular}{|l|r|r|r|r|}
\hline & \multicolumn{1}{|c|}{ Sal } & \multicolumn{1}{c|}{ Halita } & \multicolumn{1}{|c|}{ Sais_Alta } & Sais_Baixa \\
\hline № Amostras & $263.299,00$ & $222.979,00$ & $33.432,00$ & $6.888,00$ \\
\hline Mínimo & $1.574,67$ & $2.859,69$ & $2.846,15$ & $3.308,63$ \\
\hline 25o Percentil & $4.479,12$ & $4.478,52$ & $4.526,52$ & $4.035,82$ \\
\hline Mediana & $4.535,87$ & $4.531,00$ & $4.791,37$ & $4.285,47$ \\
\hline 75 Percentil & $4.619,61$ & $4.607,19$ & $5.276,91$ & $4.479,17$ \\
\hline Máximo & $7.056,32$ & $6.718,69$ & $7.056,32$ & $5.776,75$ \\
\hline Média & $4.572,57$ & $4.583,06$ & $4.897,18$ & $4.286,32$ \\
\hline Desvio Padrão & 451,00 & 281,34 & 553,28 & 307,34 \\
\hline
\end{tabular}

No dado sísmico é possível se observar, dentro da seção evaporítica, a presença de três sismofácies: estratificada, homogênea (sem contraste) e caótica (figura 04).

As fortes reflexões podem ser associadas tanto aos Sais de Alta ou às rochas ígneas, no caso de amplitudes positivas, quanto aos sais de baixa (Carnalita, Taquidrita, Silvinita etc.), no caso de reflexões negativas.

Onde a sísmica fica mais lisa, ou seja, sem contraste, é associado à presença de halita. Podem ocorrer outros tipos de sais nessas regiões, contudo estes ou não foram imageados apropriadamente, ou suas espessuras ficaram abaixo do limite de detecção do método sísmico.

O dado sísmico em questão é um PSDM (pre stack depth migration), cujo dado de campo foi adquirido em alta definição (com uma cela de aquisição de 12,5m X 6,5m e cobertura de 80 traços).

O modelo de velocidade intervalar utilizado na migração do dado (figura 05), dentro da camada de sal, possui uma velocidade intervalar média, obtido através inversões tomográficas, por volta de $4500 \mathrm{~m} / \mathrm{s}$. O intervalo varia pouco em torno desse valor, contudo há uma tendência para velocidades maiores. Constata-se, ainda, que não há um caráter geológico nesse modelo e que, visualmente, se destacam inúmeras faixas verticais de velocidade.

Alguns cuidados devem ser tomados na utilização desta sistemática:

1) A amplitude reflete a interface e não a camada, propriamente dita;

2) O critério de resolução deve sempre ser considerado e respeitado, dado que, em geral, as ocorrências destas rochas, ditas anômalas em termos de velocidades são, raramente, maiores do que poucas dezenas de metros;

3) A presença dos lobos laterais das fortes reflexões que podem ser caracterizados, de forma equivocada, como reflexões geradas de algum material quando, na verdade são, apenas, lobos laterais da wavelet convolvida com o coeficiente de reflexão da camada.

Com os cuidados acima descritos é possível modelar a velocidade, levando-se em consideração os valores de amplitude/ impedâncias caracterizadas como respostas dos respectivos litotipos (sais/ rocha ígnea).

Testes devem ser realizados a fim de se evitar uma proporção de velocidades altas e/ou baixas muito acima das encontradas em poços.

As velocidades são geradas como se fossem informações discretas, sendo necessária uma etapa de filtragem tanto lateral como vertical no modelo, visando propiciar o caráter mais contínuo das informações geradas.

\section{Resultados}

O resultado foi um modelo de velocidade intervalar mais representativo da geologia da seção evaporítica do que o do volume de velocidade gerado pela tomografia oriundo do processamento original

Pela quantidade de poços e a perfilagem em quase todo o intervalo de ocorrência dos evaporitos, pode-se inferir uma estatística das velocidades mais robusta para a definição do intervalo de valores que seriam aplicados na modelagem de velocidade. Os perfis de litologia se mostraram inconsistentes em algumas zonas e tiveram que ser reinterpretados por uma equipe de geólogos da empresa, chegando a resultados mais confiáveis.

Cerca de $90 \%$ das litologias descritas nos poços, dentro da seção evaporítica, é composta por halita, porém a maioria fora perfurado nas regiões onde a sísmica apresenta sismofácies mais homogênea, correspondendo, de fato, à predominância desse mineral. Apenas na região dos poços 02, 03, 04 e 12 foram observadas, na sísmica, mais sismofácies características de estratificações (por exemplo, na parte esquerda da figura 04). A informação dessas regiões é de suma importância, pois ajudaria a caracterizar melhor as velocidades das zonas estratificadas e, os poços, com suas descrições litológicas e de perfis confirmam isto.

As altas velocidades do modelo (figura 06) mostraram uma boa correlação com as anidritas encontrada em poços, contudo as finas camadas por estarem abaixo da resolução máxima da sísmica não foram incorporadas.

Os sais de baixa foram pouco representativos em poços e, provavelmente, as suas ocorrências estavam abaixo do limite de detecção da sísmica e mal ocorrem no modelo.

O modelo foi usado para conversão tempo/profundidade dos horizontes dessa área, porém o resultado foi uma profundidade sempre maior do que a constatada nos poços. Pode-se concluir que a velocidade média está maior que a real, dois fatores ou a soma deles podem estar contribuindo para isso: incorporação de sais de alta acima da realidade ou os sais de baixa foram subestimados durante o trabalho.

Para corrigir esse efeito, o modelo de velocidade foi calibrado utilizando as tabelas tempo/profundidade inferidas nos poços. Para a calibração foi usado o método de kigragem com deriva externa, tendo como 
variável hard as informações do poço e o soft a velocidade média modelada da sísmica. Chegou-se assim, a horizontes com profundidades próximas das constatadas nos poços.

\section{Discussão e Conclusões}

O uso da amplitude/impedância como fator na modelagem de velocidades sísmicas carece, ainda, de mais estudos. Contudo os resultados obtidos se mostram robustos em uma série de aplicações:

1) Geração de Cenários de Incertezas através de processos de calibração Tempo x Profundidade e/ou Profundidade $\mathrm{x}$ Profundidade;

2) Reprocessamentos sísmico, em especial para os processamentos PSDM, utilizando algoritmos que necessitam modelos de velocidades mais refinados, que podem, por sua vez, auxiliar na geração de melhores resultados;

3) Estudos de iluminação que devem sempre considerar a natureza geológica na qual os raios deverão ser propagados para simular a resposta mais real;

4) Modelagem geomecânica, que se beneficia da representação mais refinada do intervalo salífero.

Um importante teste que está em andamento é a utilização dessa velocidade na construção do modelo de baixa frequência aliado a informação de poços que servirá de insumo para uma inversão sísmica determinística. Caso o resultado seja satisfatório, os cubos de impedâncias servirão como entrada para a construção de um novo modelo de velocidade.

Este fluxograma está em franco desenvolvimento e novas funcionalidades estão sendo inseridas visando gerar ainda mais robustez às técnicas e resultados obtidos.

\section{Agradecimentos}

Os autores agradecem a toda equipe de reservatório envolvida no projeto e a Petrobras por permitir a divulgação desse trabalho.

\section{Referências}

Amaral, P.J., Maul, A., Falcão, L., González, M. \& González, G., 2015. Estudo Estatístico da Velocidade dos Sais na Camada Evaporítica na Bacia de Santos. International Congress of the Brazilian Geophysical Society, 14, Rio de Janeiro - RJ.

Fetter, M., Daltro, D., Lafferrieire, F. \& Souza JR., O. G., 2004. Filtragem de Velocidades Intervalares Através de Krigagem Fatorial. SIMGEF (Simpósio de Geofísica da Petrobras). Aracajú - SE.

González, M., Gobatto, F.; Maul, A., Falcão, L., Amaral, P.J., Oliveira, L., Meneguim, T. 2016. Proposed Workflow to Incorporate Stratification within Salt Section using Velocity and Seismic Attributes. Third EAGE/SBGf
Workshop 2016 - Quantitative Seismic Interpretation of Lacustrine Carbonates, Rio de Janeiro -RJ

Jardim, F., Maul, A., Falcão, L. \& González, G. 2015. Estimating Amplitude Uncertainties through Illumination Studies for a Pre-salt Reservoir. International Congress of the Brazilian Geophysical Society, 14, Rio de Janeiro RJ.

Maul, A., Jardim, F., Falcão, L., \& González, G., 2015. Observing Amplitude Uncertainties for a Pre-salt Reservoir Using Illumination Study (Hit-maps). EAGE Conference and Exhibition 2015, 77, Madrid - Spain.

Maul, A., Falcão, L., Gobatto, F.; Novellino, V., Jardim, F., Oliveira, L., Meneguim, T., Amaral, P., Borges, F., Teixeira, L., Monteiro, R., González, G., González, M. 2016 Incorporação de Estratificações dentro da Seção Evaporítica Utilizando Velocidades e Atributos Sísmicos. INFORME Geofísico Petrobras, 152, Rio de Janeiro - RJ

Meneguim, T., Mendes, S., Maul, A., Falcão, L., González, M. \& González, G. 2015. Combining Seismic Facies Analysis and Well Information to Guide New Interval Velocity Models for a Pre-Salt Study, Santos Basin, Brazil. International Congress of the Brazilian Geophysical Society, 14, Rio de Janeiro - RJ.

Oliveira, L., Falcão, L., Maul, A., Rosseto, J., González, M. \& González, G. 2015b. Geological Velocity Approach in Order to Obtain a Detailed Velocity Model for the Evaporitic Section, Santos Basin, Brazil.International Congress of the Brazilian Geophysical Society, 14, Rio de Janeiro - RJ.

Virieux, J., Operto, S., 2009. An overview of full-waveform inversion in exploration geophysics. GEOPHYSICS, VOL. 74 , NO. 6 


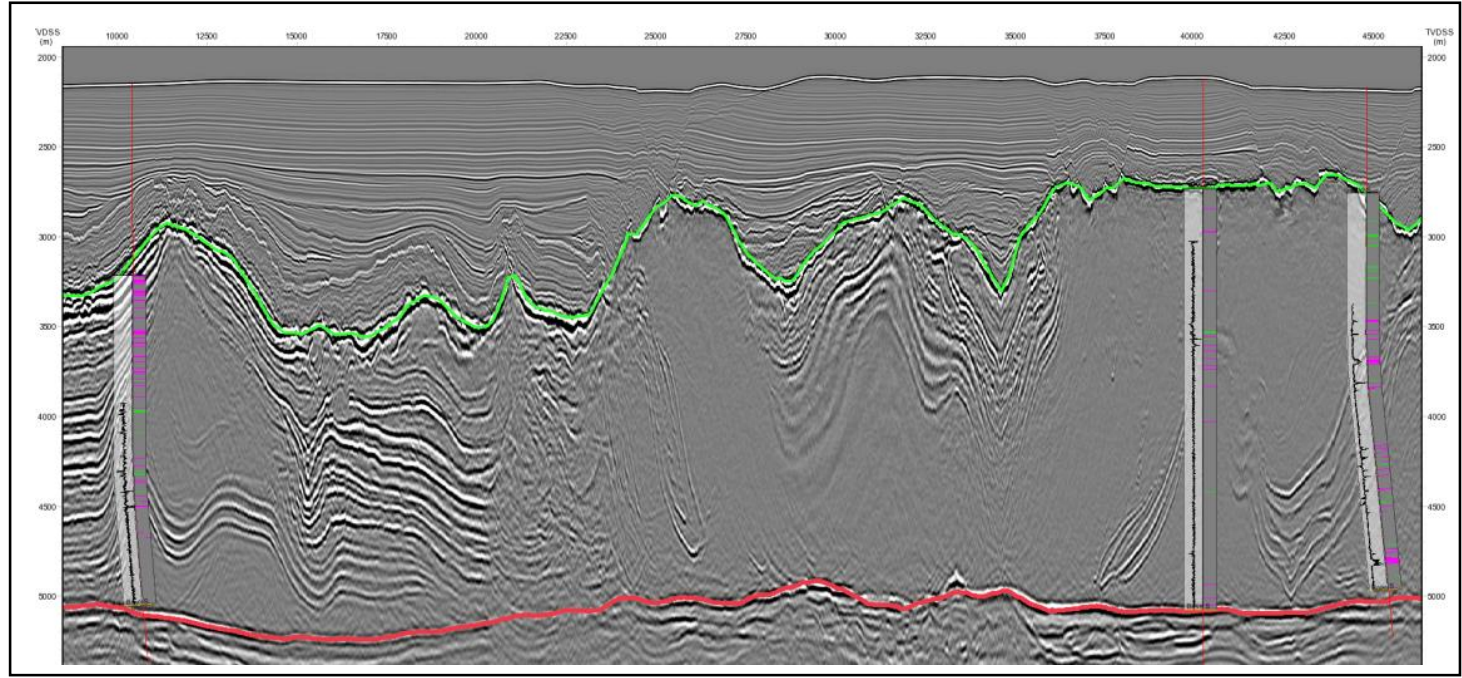

Figura 04 - Sísmica em profundidade contendo alguns perfis litológicos dos poços perfurados na área. Notar que há uma certa correlação entre as reflexões e a ocorrência ou do sal de baixa ou de alta

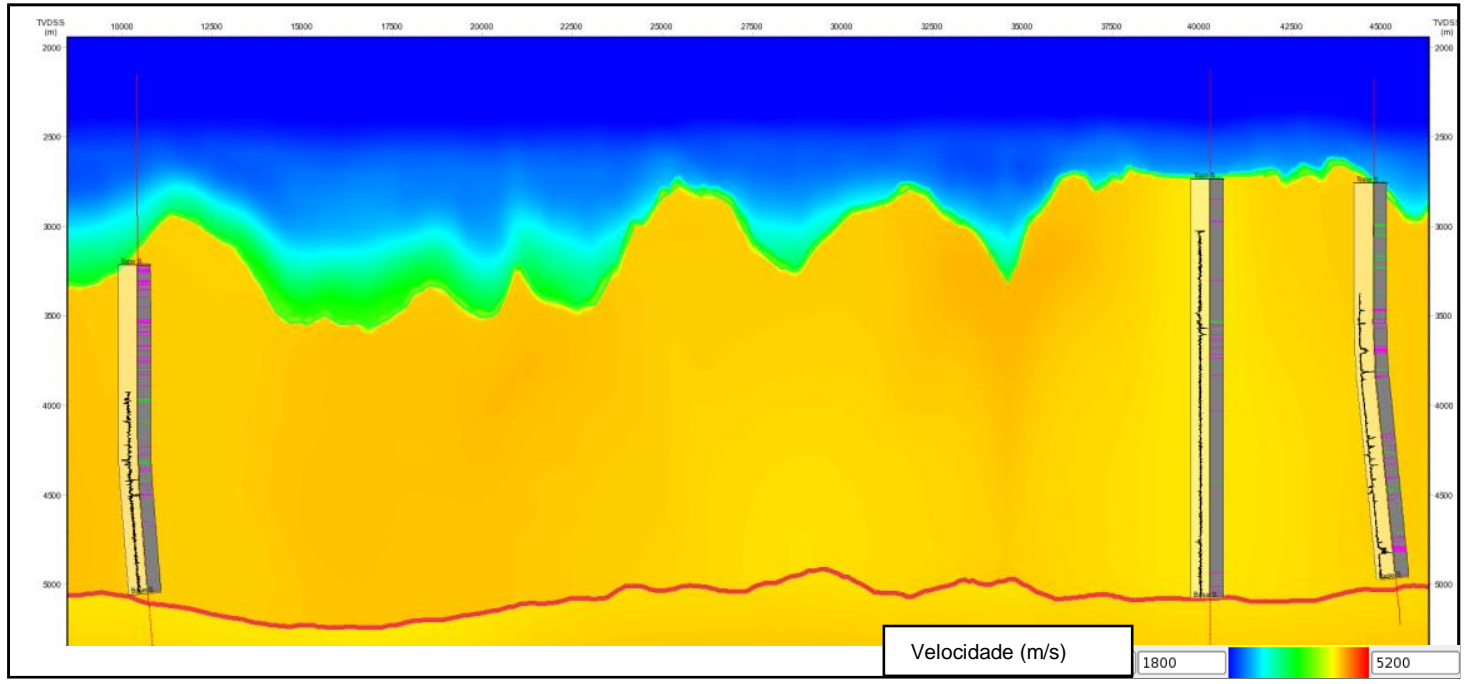

Figura 05 - Cubo de velocidade intervalar originário da inversão tomográfica

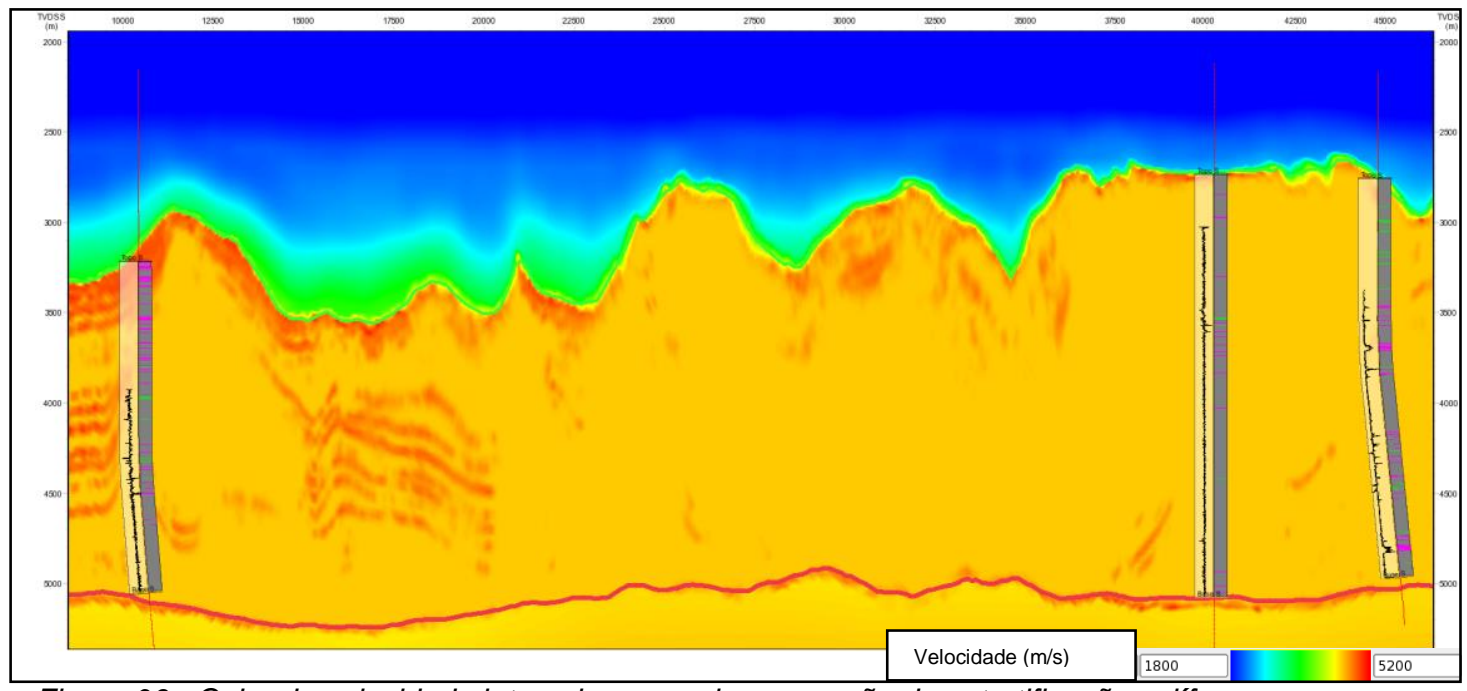

Figura 06 - Cubo de velocidade intervalar com a incorporação da estratificação salífera. 